\title{
PSR J1906+0722: AN ELUSIVE GAMMA-RAY PULSAR
}

C. J. Clark ${ }^{1,2}$, H. J. Pletsch ${ }^{1,2}$, J. Wu ${ }^{3}$, L. Guillemot ${ }^{4,5}$, M. AckermanN ${ }^{6}$, B. Allen ${ }^{1,2,7}$, A. De Angelis ${ }^{8}$, C. Aulbert ${ }^{1,2}$,

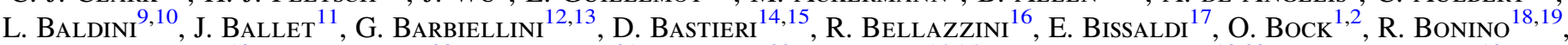
E. Bottacini ${ }^{10}$, T. J. Brandt ${ }^{20}$, J. Bregeon ${ }^{21}$, P. Bruel ${ }^{22}$, S. Buson ${ }^{14,15}$, G. A. Caliandro ${ }^{10,23}$, R. A. Cameron ${ }^{10}$, M. Caragiulo ${ }^{17}$, P. A. Caraveo ${ }^{24}$, C. Cecchi ${ }^{25,26}$, D. J. Champion ${ }^{3}$, E. Charles ${ }^{10}$, A. CheKhtman ${ }^{27,62}$, J. Chiang ${ }^{10}$, G. Chiaro $^{15}$, S. Ciprini ${ }^{25,28,29}$, R. Claus ${ }^{10}$, J. Cohen-Tanugi ${ }^{21}$, A. Cuéllar ${ }^{1,2}$, S. Cutini ${ }^{25,28,29}$, F. D’Ammando ${ }^{30,31}$, R. Desiante ${ }^{18,32}$, P. S. Drell ${ }^{10}$, H. B. Eggenstein ${ }^{1,2}$, C. Favuzzi ${ }^{17,33}$, H. FehrmanN ${ }^{1,2}$, E. C. Ferrara ${ }^{20}$, W. B. Focke ${ }^{10}$, A. Franckowiak $^{10}$, P. Fusco ${ }^{17,33}$, F. Gargano ${ }^{17}$, D. Gasparrini ${ }^{25,28,29}$, N. Giglietto ${ }^{17,33}$, F. Giordano ${ }^{17,33}$, T. Glanzman ${ }^{10}$, G. Godfrey $^{10}$, I. A. Grenier ${ }^{11}$, J. E. Grove ${ }^{34}$, S. Guiriec ${ }^{20,63}$, A. K. Harding ${ }^{20}$, E. Hays ${ }^{20}$, J. W. HeWITT ${ }^{35,36}$, A. B. Hill ${ }^{10,37}$,

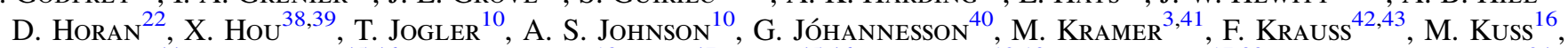

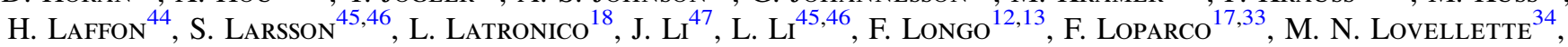
P. Lubrano $^{25,26}$, B. Machenschalk ${ }^{1,2}$, A. Manfreda ${ }^{16}$, M. Marelli $^{24}$, M. Mayer ${ }^{6}$, M. N. Mazziotta ${ }^{17}$, P. F. Michelson ${ }^{10}$,

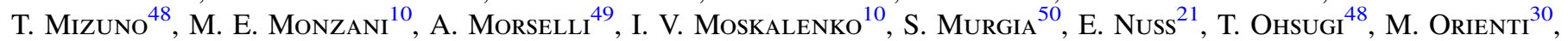
E. Orlando ${ }^{10}$, F. de Palma ${ }^{17,51}$, D. Paneque ${ }^{10,52}$, M. Pesce-Rollins ${ }^{10,16}$, F. Piron ${ }^{21}$, G. Pivato ${ }^{16}$, S. Rainò ${ }^{17,33}$,

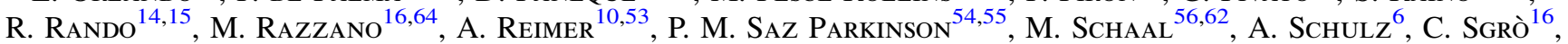
E. J. Siskind ${ }^{57}$, F. Spada ${ }^{16}$, G. Spandre ${ }^{16}$, P. Spinelli ${ }^{17,33}$, D. J. Suson ${ }^{58}$, H. Takahashi ${ }^{59}$, J. B. Thayer ${ }^{10}$, L. Tibaldo ${ }^{10}$, P. Torne ${ }^{3}$, D. F. Torres ${ }^{47,60}$, G. Tosti ${ }^{25,26}$, E. Troja ${ }^{20,61}$, G. Vianello ${ }^{10}$, K. S. Wood ${ }^{34}$, M. WoOd ${ }^{10}$, And M. YASSine ${ }^{21}$ ${ }^{1}$ Albert-Einstein-Institut, Max-Planck-Institut für Gravitationsphysik, D-30167 Hannover, Germany; colin.clark@ @aei.mpg.de

${ }^{2}$ Leibniz Universität Hannover, D-30167 Hannover, Germany

${ }^{3}$ Max-Planck-Institut für Radioastronomie, Auf dem Hügel 69, D-53121 Bonn, Germany

${ }^{4}$ Laboratoire de Physique et Chimie de l'Environnement et de l'Espace-Université d'Orléans/CNRS, F-45071 Orléans Cedex 02, France

${ }^{5}$ Station de radioastronomie de Nançay, Observatoire de Paris, CNRS/INSU, F-18330 Nançay, France

${ }^{6}$ Deutsches Elektronen Synchrotron DESY, D-15738 Zeuthen, Germany

${ }^{7}$ Department of Physics, University of Wisconsin-Milwaukee, P.O. Box 413, Milwaukee, WI 53201, USA

${ }^{8}$ Dipartimento di Fisica, Università di Udine and Istituto Nazionale di Fisica Nucleare, Sezione di Trieste, Gruppo Collegato di Udine, I-33100, Udine, Italy

${ }^{9}$ Università di Pisa and Istituto Nazionale di Fisica Nucleare, Sezione di Pisa I-56127 Pisa, Italy

${ }^{10}$ W. W. Hansen Experimental Physics Laboratory, Kavli Institute for Particle Astrophysics and Cosmology, Department of Physics and SLAC National Accelerator Laboratory, Stanford University, Stanford, CA 94305, USA

${ }^{11}$ Laboratoire AIM, CEA-IRFU/CNRS/Université Paris Diderot, Service d'Astrophysique, CEA Saclay, F-91191 Gif sur Yvette, France

${ }^{12}$ Istituto Nazionale di Fisica Nucleare, Sezione di Trieste, I-34127 Trieste, Italy

${ }^{13}$ Dipartimento di Fisica, Università di Trieste, I-34127 Trieste, Italy

${ }^{14}$ Istituto Nazionale di Fisica Nucleare, Sezione di Padova, I-35131 Padova, Italy

${ }^{15}$ Dipartimento di Fisica e Astronomia “G. Galilei," Università di Padova, I-35131 Padova, Italy

${ }_{17}^{16}$ Istituto Nazionale di Fisica Nucleare, Sezione di Pisa, I-56127 Pisa, Italy

${ }^{17}$ Istituto Nazionale di Fisica Nucleare, Sezione di Bari, I-70126 Bari, Italy

${ }^{18}$ Istituto Nazionale di Fisica Nucleare, Sezione di Torino, I-10125 Torino, Italy

${ }^{19}$ Dipartimento di Fisica Generale "Amadeo Avogadro," Università degli Studi di Torino, I-10125 Torino, Italy

${ }^{20}$ NASA Goddard Space Flight Center, Greenbelt, MD 20771, USA

${ }^{21}$ Laboratoire Univers et Particules de Montpellier, Université Montpellier, CNRS/IN2P3, Montpellier, France

${ }^{22}$ Laboratoire Leprince-Ringuet, École polytechnique, CNRS/IN2P3, Palaiseau, France

${ }^{23}$ Consorzio Interuniversitario per la Fisica Spaziale (CIFS), I-10133 Torino, Italy

${ }^{24}$ INAF-Istituto di Astrofisica Spaziale e Fisica Cosmica, I-20133 Milano, Italy

${ }_{25}^{25}$ Istituto Nazionale di Fisica Nucleare, Sezione di Perugia, I-06123 Perugia, Italy

${ }^{26}$ Dipartimento di Fisica, Università degli Studi di Perugia, I-06123 Perugia, Italy

${ }^{27}$ College of Science, George Mason University, Fairfax, VA 22030, USA

28 Agenzia Spaziale Italiana (ASI) Science Data Center, I-00133 Roma, Italy

${ }^{29}$ INAF Osservatorio Astronomico di Roma, I-00040 Monte Porzio Catone (Roma), Italy

${ }^{30}$ INAF Istituto di Radioastronomia, I-40129 Bologna, Italy

${ }^{31}$ Dipartimento di Astronomia, Università di Bologna, I-40127 Bologna, Italy

${ }^{32}$ Università di Udine, I-33100 Udine, Italy

${ }^{33}$ Dipartimento di Fisica "M. Merlin" dell’Università e del Politecnico di Bari, I-70126 Bari, Italy

${ }^{34}$ Space Science Division, Naval Research Laboratory, Washington, DC 20375-5352, USA

${ }^{35}$ Department of Physics and Center for Space Sciences and Technology, University of Maryland Baltimore County, Baltimore, MD 21250, USA

${ }^{36}$ Center for Research and Exploration in Space Science and Technology (CRESST) and NASA Goddard Space Flight Center, Greenbelt, MD 20771, USA

${ }^{37}$ School of Physics and Astronomy, University of Southampton, Highfield, Southampton, SO17 1BJ, UK

${ }^{38}$ Yunnan Observatories, Chinese Academy of Sciences, Kunming 650216, China

${ }^{39}$ Key Laboratory for the Structure and Evolution of Celestial Objects, Chinese Academy of Sciences, Kunming 650216, China

${ }^{40}$ Science Institute, University of Iceland, IS-107 Reykjavik, Iceland

${ }^{41}$ Jodrell Bank Centre for Astrophysics, School of Physics and Astronomy, The University of Manchester, M13 9PL, UK

${ }^{42}$ Dr. Remeis Sternwarte \& ECAP, Universität Erlangen-Nürnberg, Sternwartstrasse 7, D-96049 Bamberg, Germany

${ }^{43}$ Institut für Theoretische Physik und Astrophysik, Universität Würzburg, Emil-Fischer-Str. 3, D-97074 Würzburg, Germany

${ }^{44}$ Centre d'Études Nucléaires de Bordeaux Gradignan, IN2P3/CNRS, Université Bordeaux 1, BP120, F-33175 Gradignan Cedex, France

${ }^{45}$ Department of Physics, KTH Royal Institute of Technology, AlbaNova, SE-106 91 Stockholm, Sweden

${ }^{46}$ The Oskar Klein Centre for Cosmoparticle Physics, AlbaNova, SE-106 91 Stockholm, Sweden

${ }^{47}$ Institute of Space Sciences (IEEC-CSIC), Campus UAB, E-08193 Barcelona, Spain

${ }^{48}$ Hiroshima Astrophysical Science Center, Hiroshima University, Higashi-Hiroshima, Hiroshima 739-8526, Japan

${ }^{49}$ Istituto Nazionale di Fisica Nucleare, Sezione di Roma "Tor Vergata," I-00133 Roma, Italy 


\author{
${ }^{50}$ Center for Cosmology, Physics and Astronomy Department, University of California, Irvine, CA 92697-2575, USA \\ ${ }^{51}$ Università Telematica Pegaso, Piazza Trieste e Trento, 48, I-80132 Napoli, Italy \\ 52 Max-Planck-Institut für Physik, D-80805 München, Germany \\ ${ }^{53}$ Institut für Astro- und Teilchenphysik and Institut für Theoretische Physik, Leopold-Franzens-Universität Innsbruck, A-6020 Innsbruck, Austria \\ ${ }^{54}$ Santa Cruz Institute for Particle Physics, Department of Physics and Department of Astronomy and Astrophysics, \\ University of California at Santa Cruz, Santa Cruz, CA 95064, USA \\ ${ }^{55}$ Department of Physics, The University of Hong Kong, Pokfulam Road, Hong Kong, China \\ ${ }^{56}$ National Research Council Research Associate, National Academy of Sciences, Washington, DC 20001 \\ ${ }^{57}$ NYCB Real-Time Computing Inc., Lattingtown, NY 11560-1025, USA \\ ${ }^{58}$ Department of Chemistry and Physics, Purdue University Calumet, Hammond, IN 46323-2094, USA \\ ${ }^{59}$ Department of Physical Sciences, Hiroshima University, Higashi-Hiroshima, Hiroshima 739-8526, Japan \\ ${ }^{60}$ Institució Catalana de Recerca i Estudis Avançats (ICREA), Barcelona, Spain \\ ${ }^{61}$ Department of Physics and Department of Astronomy, University of Maryland, College Park, MD 20742, USA \\ Received 2015 June 24; accepted 2015 July 15; published 2015 August 4
}

\begin{abstract}
We report the discovery of PSR J1906+0722, a gamma-ray pulsar detected as part of a blind survey of unidentified Fermi Large Area Telescope (LAT) sources being carried out on the volunteer distributed computing system, Einstein@Home. This newly discovered pulsar previously appeared as the most significant remaining unidentified gamma-ray source without a known association in the second Fermi-LAT source catalog (2FGL) and was among the top 10 most significant unassociated sources in the recent third catalog (3FGL). PSR J1906+0722 is a young, energetic, isolated pulsar, with a spin frequency of $8.9 \mathrm{~Hz}$, a characteristic age of $49 \mathrm{kyr}$, and spin-down power $1.0 \times 10^{36} \mathrm{erg} \mathrm{s}^{-1}$. In 2009 August it suffered one of the largest glitches detected from a gamma-ray pulsar $\left(\Delta f / f \approx 4.5 \times 10^{-6}\right)$. Remaining undetected in dedicated radio follow-up observations, the pulsar is likely radio-quiet. An off-pulse analysis of the gamma-ray flux from the location of PSR J1906+0722 revealed the presence of an additional nearby source, which may be emission from the interaction between a neighboring supernova remnant and a molecular cloud. We discuss possible effects which may have hindered the detection of PSR J1906+0722 in previous searches and describe the methods by which these effects were mitigated in this survey. We also demonstrate the use of advanced timing methods for estimating the positional, spin and glitch parameters of difficult-to-time pulsars such as this.
\end{abstract}

Key words: gamma rays: stars - pulsars: individual (PSR J1906+0722)

\section{INTRODUCTION}

The large collecting area and continuous observation mode of the Fermi Large Area Telescope (LAT; Atwood et al. 2009) make it an ideal instrument for the detection and analysis of periodic gamma-ray emission from pulsars. Through the careful analysis of the arrival times of photons covering the 6 years since its launch, the LAT has discovered pulsed gammaray emission from more than 160 pulsars $^{65}$ (Abdo et al. 2013; Caraveo 2014).

While the majority of these pulsars were first found in radio observations (e.g., Abdo et al. 2009b, 2009c), the ephemerides from which could be used to test for gamma-ray pulsations, a substantial fraction of the gamma-ray pulsar population was discovered through blind searches of Fermi-LAT data (e.g., Abdo et al. 2009a; Saz Parkinson et al. 2010).

In a recent work (Pletsch \& Clark 2014) we presented newly advanced methods designed to increase the sensitivity of blind searches without increasing the computational cost. These improvements have since been incorporated into a new blind survey of unidentified, pulsar-like Fermi-LAT sources being conducted on the distributed volunteer computing system, Einstein@Home. ${ }^{66}$ Previous surveys have been extremely successful in detecting new gamma-ray pulsars (Pletsch et al. 2012a, 2012b; Pletsch et al. 2013), and the

\footnotetext{
${ }^{62}$ Resident at Naval Research Laboratory, Washington, DC 20375, USA.

63 NASA Postdoctoral Program Fellow, USA.

64 Funded by contract FIRB-2012-RBFR12PM1F from the Italian Ministry of Education, University and Research (MIUR).

65 http://tinyurl.com/fermipulsars

66 http://www.einsteinathome.org
}

newly improved search methods, in combination with the latest Fermi-LAT data, offer a significant increase in sensitivity.

As part of this survey, we carried out a blind search for pulsed emission from a point source in the third Fermi-LAT source catalog (3FGL, Acero et al. 2015), 3FGL J1906.6+0720. This source, previously known as 2FGL J1906.5+0720 (Nolan et al. 2012), is highly significant and stands out as the most significant unassociated 2FGL source. Moreover, it was included in the "bright" pulsar-like source list described by Romani (2012). An investigation of the spectral properties of $2 \mathrm{FGL}$ sources found that, after the source associated with the Galactic Center, 2FGL J1906.5+0720 was the unidentified source most likely to contain a pulsar (Lee et al. 2012). As such, over recent years, this source has been searched for pulsations, both in gamma-rays (e.g., Pletsch et al. 2012a; Xing \& Wang 2014) and in radio observations (e.g., Barr et al. 2013). However, despite these attempts, pulsed emission from this source remained undetected until now.

Here, we present the discovery and follow-up study of PSR J1906+0722, a young isolated gamma-ray pulsar detected by the Einstein@Home survey.

\section{DISCOVERY}

\subsection{Data Preparation}

In the blind search we analyzed Fermi-LAT data recorded between 2008 August 4 and 2014 April 6. The Fermi Science Tools ${ }^{67}$ were used to extract Pass 8 source class photons, which were analyzed using the P8_SOURCE_V3 instrument response

\footnotetext{
${ }^{67}$ http://fermi.gsfc.nasa.gov/ssc/data/analysis/software
} 
functions (IRFs). ${ }^{68}$ We used gt select to select photons with reconstructed directions within an $8^{\circ}$ region of interest (ROI) around 3FGL J1906.6+0720, photon energies $>100 \mathrm{MeV}$ and zenith angles $<100^{\circ}$. We only included photons detected when the LAT was working in normal science mode, and with rocking angle $<52^{\circ}$.

To assign photon weights representing the probability of each photon having been emitted by the target source (Kerr 2011), we performed a likelihood spectral analysis using the pointlike package. We built a source model by including all 3FGL catalog sources located within $13^{\circ}$ of 3FGL J1906.6+0720, while allowing the spectral parameters of point sources within $5^{\circ}$ to vary. We modeled the gamma-ray spectrum of this source with an exponentially cutoff power law, typical of gamma-ray pulsar spectra (Nolan et al. 2012). We used the template_4years_P8_V2_scaled.fits map cube and isotropic_source_4years_P8V3 template to model the Galactic diffuse emission and the isotropic diffuse background respectively. ${ }^{69}$ The normalization parameters of both diffuse components were left free. Finally, the photon weights were computed using gtsrcprob, based on the bestfit source model resulting from the likelihood analysis.

\subsection{Blind Search Method}

For the blind search, we assumed a canonical isolated pulsar model, making it necessary to search in four parameters: spin frequency, $f$, spin-down rate, $\dot{f}$, R.A., $\alpha$ and decl., $\delta$.

The basis for most blind searches for gamma-ray pulsars is the well-known multistage scheme based around an initial semicoherent search (e.g., Atwood et al. 2006; Pletsch et al. 2012a). For this survey, we implemented the form of the multistage search scheme described in Pletsch \& Clark (2014), where the initial semicoherent stage uses a lag-window of duration $2^{21} \mathrm{~s} \approx 24$ days.

Notably, this survey incorporates an intermediate semicoherent refinement step, with a longer (more sensitive) lagwindow of $2^{22} \mathrm{~s} \approx 48$ days, reducing the parameter space around each first-stage candidate to be searched in the final fullycoherent follow-up step. This improves the efficiency of the follow-up stage, and allows the search to "walk" away (in all four search parameters) from the original location of the candidate if necessary.

Figure 1 illustrates the importance of these new techniques. In the blind survey, we searched a conservatively large circular region around the 3FGL sky location with a radius 50\% larger than the 3FGL 95\% confidence region. As evident from Figure 1, the pulsar lies far outside the original source's confidence region, and also outside our search region. We therefore owe its detection to the large resolution of the semicoherent step, and the flexibility of the follow-up steps, which allow for signals to be detected despite a large offset between the signal parameters and the search location.

The most significant pulsar candidates from the blind search were automatically refined using the $H$-test statistic (de Jager et al. 1989). This revealed an interesting candidate; however the measured signal-to-noise ratio $(\mathrm{S} / \mathrm{N})$ was slightly below the detection threshold for a blind search involving a very high

\footnotetext{
${ }^{68}$ The Science Tools, IRFs and diffuse models used here are internal prerelease versions of the Pass 8 data analysis. Our results did not change substantially with the final release versions.

${ }^{69}$ http://fermi.gsfc.nasa.gov/ssc/data/access/lat/BackgroundModels.html
}

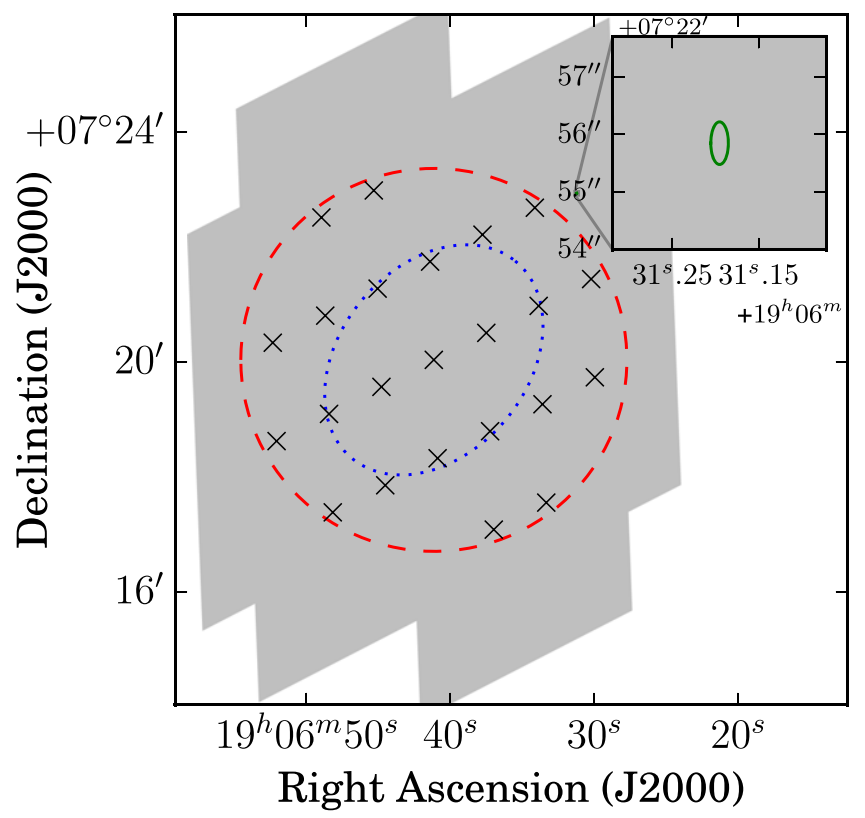

Figure 1. Sky location of PSR J1906+0722 and positional offset from the catalog location. The dotted ellipse shows the 3FGL 95\% confidence region. The dashed ellipse shows the region in which the search grid (crosses) was constructed for the initial semicoherent search stage. The filled area shows the region that can be reached by the follow-up stage as it moves away from the initial candidate location. The $1 \sigma$ region from the timing solution, shown by the solid ellipse, is highlighted in the inset.

number of trials. Upon manual inspection, clear pulsations were observed in the photon data after April 2010; however the phase of these pulsations was not constant, and exhibited "wraps" in which the pulsation phase quickly jumped by one full rotation. These features indicated that the canonical isolated pulsar model used for the blind survey was insufficient, and hence follow-up studies were required to describe the pulsar's rotation over the entire data set.

\section{FOLLOW-UP ANALYSIS}

Before carrying out follow-up analyses, we extended the data set to include photons observed until 2014 October 1 and increased the ROI to $10^{\circ}$. To speed up the timing procedure computations, we discarded photons with a probability weight below $5 \%$.

\subsection{Glitch Identification}

Since pulsations were initially only detected during the final 4 years of data, the first step was to identify any glitches within the observation time. To achieve this, we searched the local $\{f, \dot{f}\}$ space around the observed signal, in 150 day segments with approximately $90 \%$ overlap, using the $Q_{M}$-test (Bickel et al. 2008; Pletsch \& Clark 2014),

$$
Q_{M}=2 M \frac{\sum_{n=1}^{M}\left|\alpha_{n}\right|^{2} \mathcal{P}_{n}}{\sum_{n=1}^{M}\left|\alpha_{n}\right|^{2}},
$$

where $\alpha_{n}$ and $\mathcal{P}_{n}$ are the Fourier coefficients of the measured pulse profile and the coherent power at the $n$th harmonic respectively. Using the $Q_{M}$ test method to weight the contributions from each harmonic, as opposed to the commonly used $H$-test, offers a significant sensitivity improvement 

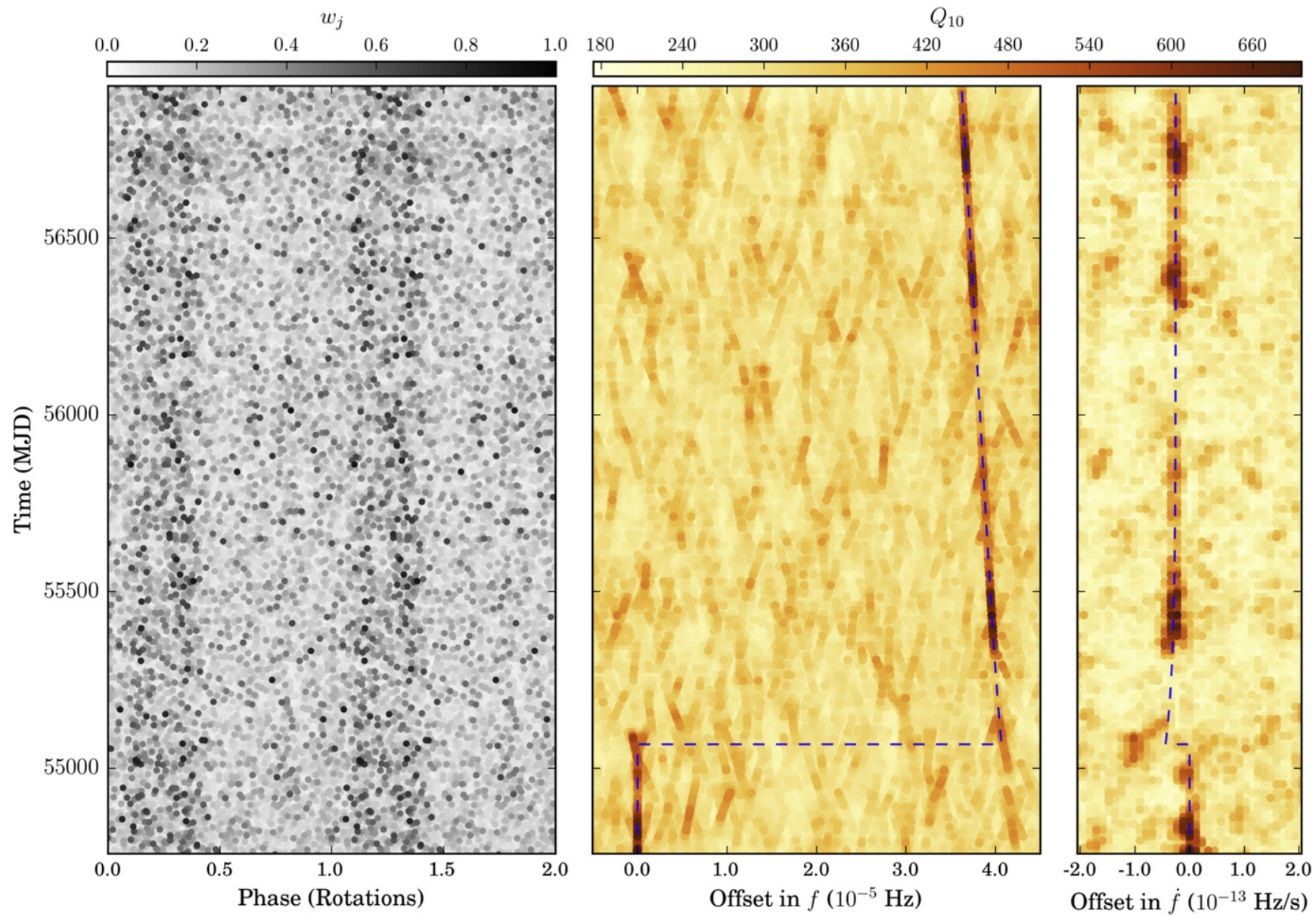

Figure 2. Evolution of the PSR J1906+0722 signal including the glitch at MJD 55067. Left: Phase-time diagram where each point represents one photon, with the intensity representing the photon weight. Center and right: The $Q_{10}$-test (shown by the color bar) calculated over small ranges in $\{f, \dot{f}\}$, centered on the pre-glitch parameters, in overlapping 150 day segments, and maximized over $\dot{f}$ and $f$ respectively. The dashed line indicates the maximum likelihood timing solution.

(Beran 1969), making it particularly useful when analyzing weak pulsar signals. For this step, we included the first 10 Fourier coefficients with appreciable power from a segment of the data in which the signal was reasonably stable. Using the results of this scan, shown in Figure 2, an initial ephemeris was produced for the timing procedure described in the following section.

\subsection{Timing Analysis}

To accurately estimate the pulsar's rotational, glitch and sky location parameters we used a variation of the timing method used by Ray et al. (2011), based on unbinned likelihood maximization. For all $N$ photons in the data set, with weights $\left\{w_{j}\right\}$, we assigned a rotational phase $\phi \equiv \phi\left(t_{j}, \boldsymbol{u}\right)$, determined by the photon's arrival time, $t_{j}$ and the set of model parameters, denoted by the vector $\boldsymbol{u}$. For a template pulse profile, $F(\phi)$, the likelihood is

$$
\mathcal{L}(\boldsymbol{u})=\prod_{j=1}^{N}\left[w_{j} F\left(\phi\left(t_{j}, \boldsymbol{u}\right)\right)+\left(1-w_{j}\right)\right] .
$$

We first constructed a template pulse profile from the (background subtracted, see Figure 3) photons within a subsection of the data set in which the initial ephemeris was believed to be accurate. When timing PSR J1906+0722 we used a template pulse profile consisting of three wrapped Gaussian functions (Abdo et al. 2013), which were fit by maximizing the likelihood within the segment.

With a template profile at hand, we then estimated the pulsar's parameters (given in Table 1) by varying them around their initial estimate to maximize the likelihood over the entire data set. The result is a likelihood maximization which is unbinned in both phase (via the template profile) and time. This avoids the need to construct a set of data subsegments for pulse time of arrival (TOA) determination. This is especially beneficial for faint pulsars, which require longer subsegments (and hence fewer TOAs) to ensure the S/N is large enough in each for accurate TOA measurement. Subsequently, using the most likely parameters, the template profile was updated and the process was iterated to maximize the overall likelihood.

To explore the multi-dimensional parameter space we used the MultiNest nested sampling algorithm (Feroz et al. 2013), which offers high sampling efficiency, and allows posterior distributions to be calculated as a by-product.

The timing procedure was carried out in two stages: first, all timing parameters were allowed to vary. Due to the shortness of the pre-glitch segment, the uncertainties in the glitch parameters dominated those of the remaining timing parameters. We therefore fixed the glitch parameters at their 


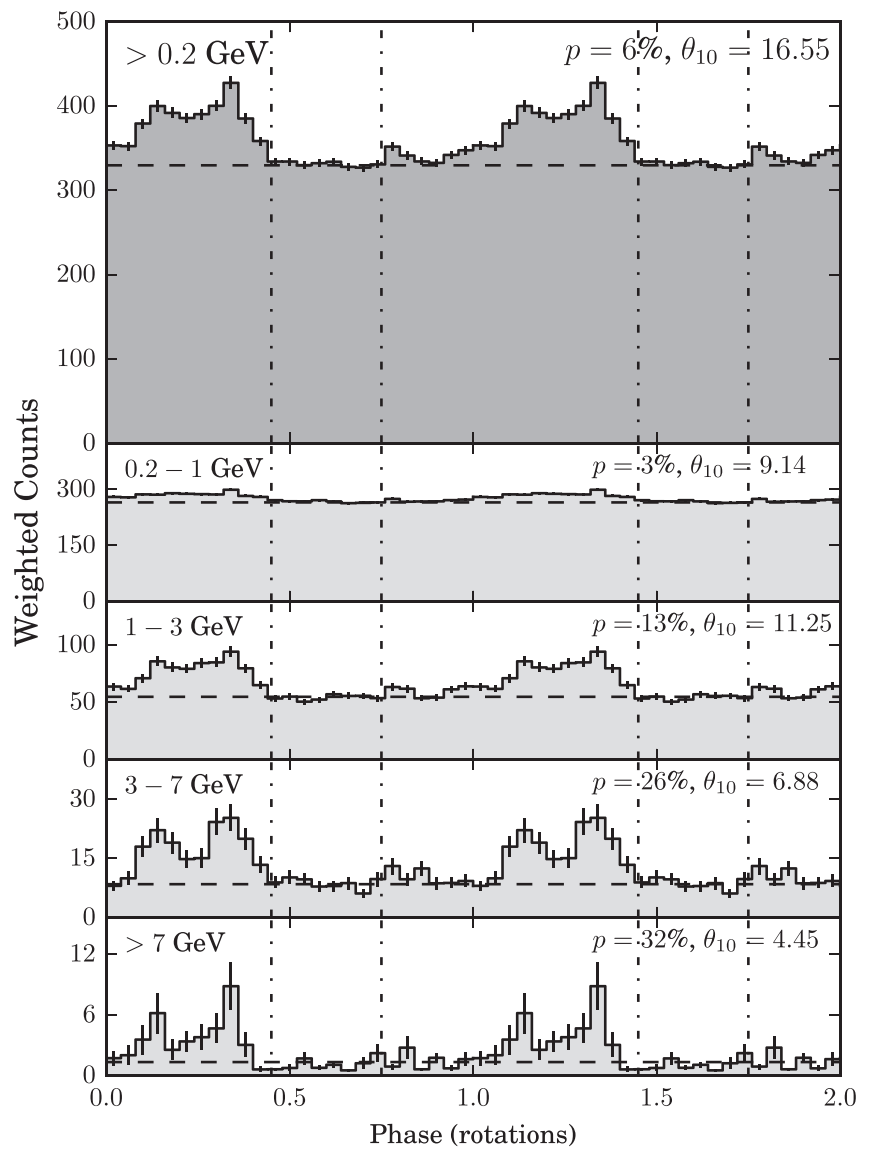

Figure 3. Top panel: weighted pulse profile of PSR J1906 +0722 given by the timing solution. Lower panels: weighted pulse profiles in increasing energy bands. In each panel, the estimated background level, calculated from the photon weights (Guillemot et al. 2012), is shown by the dashed line. The dotdashed lines mark the off-pulse phase interval used in Section 3.3. The error bars show $1 \sigma$ statistical uncertainties (Pletsch et al. 2012a). The pulsed fraction, $p$, and pulsed $\mathbf{S} / \mathrm{N}, \theta_{10}$, in each energy band is also shown.

maximum likelihood values, and fit again for the remaining timing parameters.

When timing radio pulsar glitches, Yu et al. (2013) noted that unique solutions for glitch epochs could not be found for large glitches occurring during an interval between two radio observations. We observe a similar effect here, although our limiting factor is the photon flux. When phase folding, a full rotation can be lost/gained if the offset between the model glitch epoch and the true glitch epoch is more than $1 / \Delta f \approx 0.3$ days; however, an average of only 1.4 weighted photons are observed from the pulsar within this time, making this phase wrap simply undetectable. We assumed that no phase increment occurred at the glitch, and found that the posterior distribution for the glitch epoch features several bands, separated by $1 / \Delta f$. Due to the multi-modal shape of the posterior distribution, in Table 1 we report the glitch epoch that results in the maximum likelihood and the $95 \%$ credible interval.

The inclusion of an additional nearby source in the source model and raising the energy threshold to $200 \mathrm{MeV}$ when calculating the photon weights for PSR J1906+0722 increased the $\mathrm{S} / \mathrm{N}$ (see Section 3.3). Therefore the timing analysis was repeated with the updated photon weights, and the results are given in Table 1. The time versus rotational phase diagram based on this timing solution is shown in Figure 2 and the
Table 1

Parameters for PSR J1906+0722

\begin{tabular}{lc}
\hline \hline Parameter & Value \\
\hline Range of Photon Data (MJD) & $54682-56931$ \\
Reference epoch (MJD) & 55716 \\
\hline
\end{tabular}

Timing Parameters

R.A., $\alpha(\mathrm{J} 2000.0)$

$19^{\mathrm{h}} 06^{\mathrm{m}} 31^{\mathrm{s}} .20(1)$

Decl., $\delta$ (J2000.0)

Frequency, $f(\mathrm{~Hz})$

1st frequency derivative, $\dot{f},\left(\mathrm{~Hz} \mathrm{~s}^{-1}\right)$

2nd frequency derivative, $\ddot{f},\left(\mathrm{~Hz} \mathrm{~s}^{-2}\right)$

Glitch epoch $^{\mathrm{a}}$ (MJD)

Permanent $f$ glitch increment ${ }^{\mathrm{a}}, \Delta f(\mathrm{~Hz})$

Perm. $\dot{f}$ glitch increment ${ }^{\mathrm{a}}, \Delta \dot{f}\left(\mathrm{~Hz} \mathrm{~s}^{-1}\right)$

Decaying $f$ glitch increment ${ }^{\mathrm{a}}, \Delta f_{\mathrm{d}}(\mathrm{Hz})$

$+07^{\circ} 22^{\prime} 55^{\prime \prime} 8(4)$

$8.9666688432(1)$

Glitch decay time constant ${ }^{\mathrm{a}}, \tau_{\mathrm{d}}$ (days)

$-2.884709(2) \times 10^{-12}$

$3.18(1) \times 10^{-23}$

$55067_{-9}^{+2}$

$4.033(1) \times 10^{-5}$

$-2.56(3) \times 10^{-14}$

$3.64(9) \times 10^{-7}$

$221(12)$

\begin{tabular}{|c|c|}
\hline \multicolumn{2}{|c|}{ Spectral Properties } \\
\hline Spectral index, $\Gamma$ & $1.9 \pm 0.1$ \\
\hline Cutoff energy, $E_{\mathrm{c}}(\mathrm{GeV})$ & $5.5 \pm 1.2$ \\
\hline Photon flux ${ }^{\mathrm{b}}, F_{100}$ (photons $\mathrm{cm}^{-2} \mathrm{~s}^{-1}$ ) & $(1.1 \pm 0.3) \times 10^{-7}$ \\
\hline Energy flux ${ }^{\mathrm{b}}, G_{100}\left(\mathrm{erg} \mathrm{cm}^{-2} \mathrm{~s}^{-1}\right)$ & $(7.3 \pm 1.3) \times 10^{-11}$ \\
\hline \multicolumn{2}{|c|}{ Derived Properties } \\
\hline Period, $P(\mathrm{~ms})$ & $111.524136498(1)$ \\
\hline 1st period derivative, $\dot{P}\left(\mathrm{~s} \mathrm{~s}^{-1}\right)$ & $3.587895(2) \times 10^{-14}$ \\
\hline Weighted $H$-test & 731.2 \\
\hline Characteristic age $^{\mathrm{c}}, \tau_{\mathrm{c}}(\mathrm{kyr})$ & 49.2 \\
\hline Spin-down powerc ${ }^{\mathrm{c}} \dot{E}\left(\mathrm{erg} \mathrm{s}^{-1}\right)$ & $1.02 \times 10^{36}$ \\
\hline Surface $B$-field strength ${ }^{\mathrm{c}}, B_{\mathrm{S}}(\mathrm{G})$ & $2.02 \times 10^{12}$ \\
\hline Light-cylinder $B$-field ${ }^{\mathrm{c}}, B_{\mathrm{LC}}(\mathrm{G})$ & $1.34 \times 10^{4}$ \\
\hline Heuristic distance $^{\mathrm{c}}, d_{\mathrm{h}}(\mathrm{kpc})$ & 1.91 \\
\hline
\end{tabular}

Notes. Values for timing parameters are the mean values of the marginalized posterior distributions from the timing analysis, with $1 \sigma$ uncertainties in the final digits quoted in parentheses.

${ }^{\text {a }}$ Glitch model parameters are defined in Edwards et al. (2006), with the correction noted by $\mathrm{Yu}$ et al. (2013).

${ }^{\mathrm{b}}$ Fluxes above $100 \mathrm{MeV}, F_{100}$ and $G_{100}$, were calculated by extrapolation from the $E>200 \mathrm{MeV}$ spectrum.

${ }^{c}$ Derived pulsar properties are defined in Abdo et al. (2013). The heuristic distance, $d_{\mathrm{h}}=\left(L_{\gamma}^{\mathrm{h}} / 4 \pi G_{100}\right)^{1 / 2}$, is calculated from the heuristic luminosity, $L_{\gamma}^{\mathrm{h}}$, described therein.

integrated pulse profile is displayed in Figure 3. Through these refinement and timing procedures, the initial candidate's $Q_{10^{-}}$ test S/N (Pletsch \& Clark 2014) ${ }^{70}$ was increased from $\theta_{10}=6.86$ to the highly significant value of $\theta_{10}=16.55$ given by the final timing solution.

\subsection{Off-pulse Analysis}

Fitting an exponential cutoff model to the spectrum of PSR J1906+0722 revealed a relatively high cutoff energy compared to typical gamma-ray pulsars $\left(E_{\mathrm{c}}=6.5 \pm 0.9 \mathrm{GeV}\right)$, suggesting that the spectrum could be contaminated by the presence of a nearby source as was also noted by Xing \& Wang (2014).

\footnotetext{
${ }^{70}$ The expectation value for $Q_{M}$ under the null hypothesis, $E_{0}\left[Q_{M}\right]$, in Pletsch \& Clark (2014) contains an error. S/Ns reported in this work were calculated using the corrected value of $4 M$.
} 
To investigate this possibility, we analyzed the off-pulse part of the data using photons with energies between $200 \mathrm{MeV}$ and $300 \mathrm{GeV}$. A residual test statistic (TS) map for the offpulse data (see Figure 3 ) revealed an excess $(0.28 \pm 0.02)^{\circ}$ away from PSR J1906+0722, at $(\alpha, \delta)=(286.84,7.15)$, with a TS value of 288 .

Modeling this secondary source with a power-law spectrum, we added it to the spectral model for the region, keeping its location fixed from the off-pulse analysis, but leaving its normalization and spectral index free, and analyzed again the full phase interval data. As a result, we found that the loglikelihood value increased slightly, and the new photon weights increased the $\mathrm{S} / \mathrm{N}$ of the pulsations from $\theta_{10}=16.38$ to $\theta_{10}=16.55$.

The low energy threshold of $200 \mathrm{MeV}$ was chosen to provide improved angular resolution in order to better separate the pulsar emission from that of the new source. When lower energy (100-200 MeV) photons were included in the spectral analysis, the pulsation $\mathrm{S} / \mathrm{N}$ calculated with the resulting photon weights decreased, suggesting that source confusion at low energies leads to a less reliable source model.

Figure 4 shows TS maps and spectral energy distributions for PSR J1906+0722 and the new source found in this offpulse analysis. The integrated energy flux of the secondary source above $100 \mathrm{MeV}$ is $4.34_{-0.67}^{+0.91} \times 10^{-11} \mathrm{erg} \mathrm{cm}^{-2} \mathrm{~s}^{-1}$ with a spectral index of $2.17 \pm 0.07$.

The best-fitting location of the secondary source is very close to the western edge of the supernova remnant (SNR), G41.1-0.3 (3C 397, Safi-Harb et al. 2005). Jiang et al. (2010) observed a molecular cloud interacting with the SNR at this location; it is possible that we are observing gamma-ray emission resulting from this interaction.

\section{ANALYSIS IN OTHER WAVELENGTHS}

\subsection{Radio and X-Ray Observations}

In probing for radio emission from PSR J1906+0722, we carried out a 120 minute follow-up observation with the L-band $(1.4 \mathrm{GHz})$ single-pixel receiver mounted on the $100 \mathrm{~m}$ Effelsberg Radio Telescope in Germany. The gamma-raytiming ephemeris allowed us to search the data over dispersion measure only. No evidence for radio pulsations was found. Assuming a $10 \%$ pulse width, bandwidth $\Delta F=150 \mathrm{MHz}$, telescope gain $G=1.55, n_{\mathrm{p}}=2$ polarization channels, system temperature $T_{\text {sys }}=24 \mathrm{~K}$, digitization factor $\beta=1.2$ and a signal-to-noise threshold of 5 , by the radiometer equation (Equation (A1.22), Lorimer \& Kramer 2005, p. 265), we computed a flux density limit of $\approx 21 \mu \mathrm{Jy}$. While this is below the conventional radio-quiet level of $30 \mu \mathrm{Jy}$ (Abdo et al. 2013), we note that the nearby LAT-discovered pulsar PSR J1907 +0602 has been observed in radio observations with a flux density of just $3.4 \mu \mathrm{Jy}$ (Abdo et al. 2010), and would therefore not have been detected in this radio search.

To check for a possible X-ray counterpart, we analyzed a $10 \mathrm{ks}$ observation with Swift's X-ray Telescope (Stroh \& Falcone 2013). No counterpart source was detected, with an unabsorbed-flux $(0.5-10 \mathrm{keV})$ upper limit of $2 \times 10^{-13} \mathrm{erg} \mathrm{cm}^{-2} \mathrm{~s}^{-1}$ at the pulsar position. This limit yields a gamma-ray-to-X-ray flux ratio of $>365$, or an efficiency $L_{\mathrm{X}} / \dot{E} \lesssim 8.7 \times 10^{-5}$ at distance $d_{\mathrm{h}}$, similar to other gamma-ray pulsars (Marelli et al. 2011; Pletsch et al. 2012a).

\subsection{Possible SNR Associations}

There are four known SNRs lying within $1^{\circ}$ from the timing position of PSR J1906+0722 (Green 2014). There is strong evidence that the closest of these, G41.1-0.3, is a Type Ia SNR from a Chandrasekhar mass progenitor (Yamaguchi et al. 2015), making it unlikely to be the birthplace of a pulsar. Each of the remaining nearby SNRs lies closer to other young pulsars than to PSR J1906+0722 (G41.5+0.4 and G42.0-0.1 to PSR J1906+0746; G40.5-0.5 to PSR J1907+0602), making a physical association between any of these difficult to verify. Kick-velocity requirements based on the pulsar's characteristic age and heuristic distance do not rule out any of these SNRs as the birthplace of the pulsar.

\section{DISCUSSION}

Despite several years of attempts, the identification of 2FGL J1906.5+0720 remained elusive. Now that this source has been identified as PSR J1906+0722, we here investigate potential reasons for the failure of previous searches to detect it.

Perhaps the most significant source of difficulty in the detection of PSR J1906+0722 was the large positional offset between its 3FGL catalog position and its true position. This offset, which could only be accommodated by the new followup method outlined in Section 2.2, is most likely due to the presence of the secondary source described in Section 3.3.

The close proximity of PSR J1906 +0722 to the Galactic plane ( $b=0.03)$ likely also hindered its detection, as the large majority of the weighted photons can be attributed to the background. From the pulse profile shown in Figure 3, we estimate that the pulsed fraction of the total weighted photon flux (as defined in Pletsch \& Clark 2014) is as low as 6\%. This low pulsed fraction leads to a low observable $\mathrm{S} / \mathrm{N}$, making detection more challenging.

A further complication for detecting PSR J1906+0722 was the presence of the glitch about one year into the Fermi mission. This glitch is among the largest detected from a gamma-ray pulsar in terms of relative magnitude $\left(\Delta f / f \approx 4.5 \times 10^{-6}\right)$ (Pletsch et al. 2012b). In previous searches using a shorter total observation time, the data segment after the glitch represented a much shorter fraction of the total observation time. As the time interval covered by Fermi's observations since 2008 August 1 continues to increase, the existence of a long timespan in which a pulsar's signal is stable becomes ever more likely. The increase in the weighted photon flux offered by the Pass 8 analysis (Atwood et al. 2013) further increases the observable $\mathrm{S} / \mathrm{N}$ throughout the observation time, and results in searches that are not only more sensitive overall (Laffon et al. 2015), but also more robust against glitching or noisy pulsars.

The ability to detect young gamma-ray pulsars in blind searches can be of significant importance to the overall study of energetic pulsars. For example, Ravi et al. (2010) use the observed population of radio-quiet pulsars to investigate the dependence of properties of pulsar emission geometries on the spin-down energy, $\dot{E}$. Since pulsars with a high $\dot{E}$ tend to exhibit timing noise and glitches (which do not typically affect radio searches), they are hard to find in gamma-ray data, where long integration times are required. Advanced search methods that can detect complicated signals such as that from PSR J1906+0722 are therefore crucial for reducing a potential bias against young, energetic and glitching pulsars in the 
TS
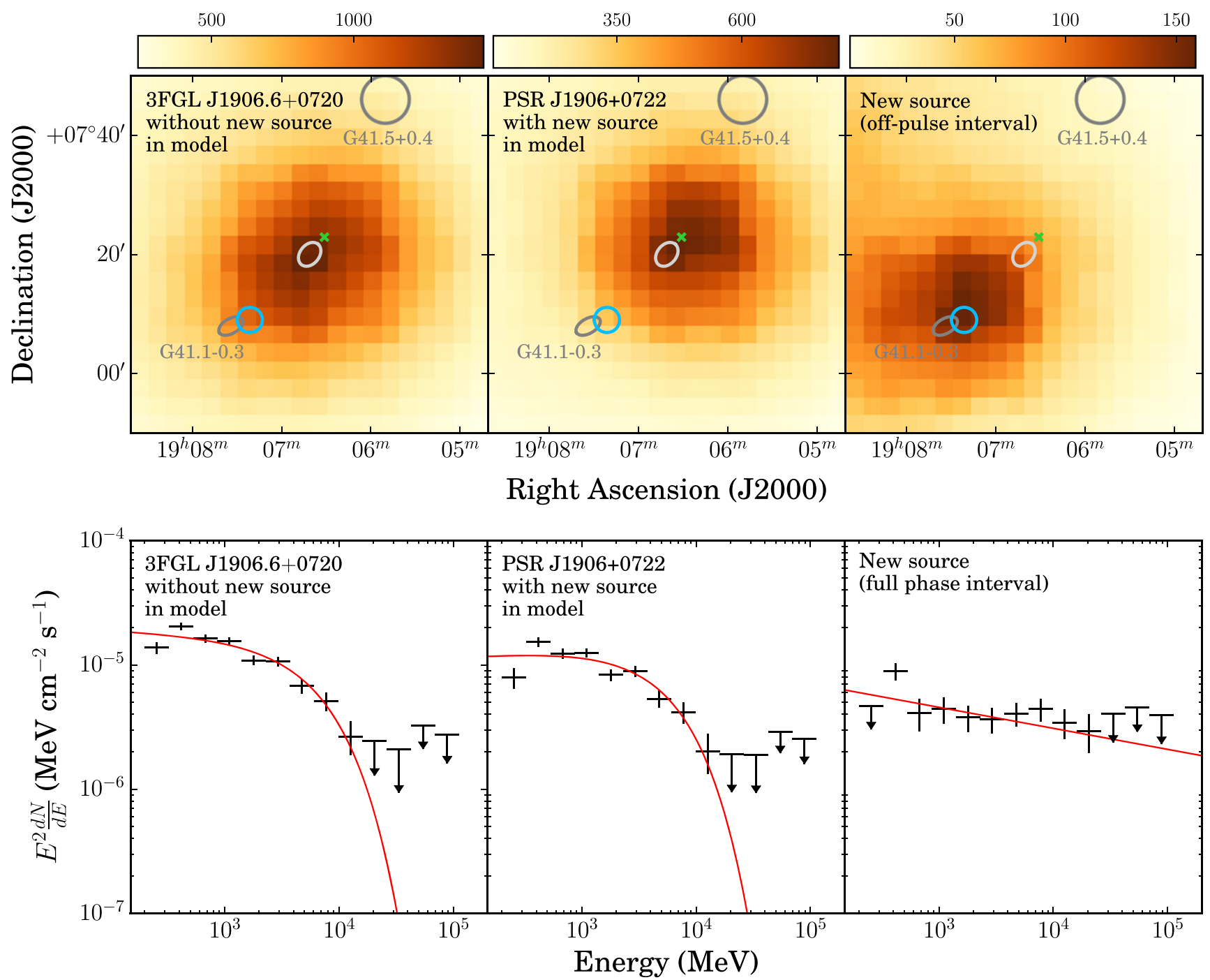

Figure 4. Top panels: test statistic (TS) sky-maps of the PSR J1906+0722 region above $200 \mathrm{MeV}$. Each pixel shows the TS value for a point source located at the pixel position. The cross represents the timing position of PSR J1906+0722. The light ellipse shows the 95\% confidence region of the 3FGL source, the blue ellipse shows the 95\% confidence region of the new secondary source, and the darker ellipses show the approximate extents of nearby SNRs. Bottom panels: Spectral energy distributions for the full-pulse interval. The solid curves present the results of the likelihood analyses of Section 3.3.

radio-quiet population. As noted by Abdo et al. (2013) and Caraveo (2014), such pulsars are indeed lacking in the Fermi pulsar sample.

This work was supported by the Max-Planck-Gesellschaft (MPG), as well as by the Deutsche Forschungsgemeinschaft (DFG) through an Emmy Noether research grant PL 710/1-1 (PI: Holger J. Pletsch). We are very grateful to all Einstein@Home volunteers who have donated their spare computing time, especially Connor Barry of Lafayette, $\mathrm{CO}$, USA and Rich Johnson of Hayward, CA, USA on whose computers PSR J1906+0722 was first detected. The Fermi-LAT Collaboration acknowledges support for LAT development, operation, and data analysis from NASA and DOE (United States); CEA/Irfu and IN2P3/CNRS (France); ASI and INFN (Italy); MEXT, KEK, and JAXA (Japan); and the K.A. Wallenberg Foundation, the Swedish Research Council, and the National Space Board (Sweden). Science analysis support in the operations phase from INAF (Italy) and CNES (France) is also gratefully acknowledged.

\section{REFERENCES}

Abdo, A. A., Ackermann, M., Ajello, M., et al. 2009a, Sci, 325, 840 Abdo, A. A., Ackermann, M., Ajello, M., et al. 2010, ApJ, 711, 64

Abdo, A. A., Ackermann, M., Atwood, W. B., et al. 2009b, ApJL, 695, L72 Abdo, A. A., Ackermann, M., Atwood, W. B., et al. 2009c, ApJ, 699, 1171 Abdo, A. A., Ajello, M., Allafort, A., et al. 2013, ApJS, 208, 17 Acero, F., Ackermann, M., Ajello, M., et al. 2015, ApJS, 218, 23

Atwood, W. B., Abdo, A. A., Ackermann, M., et al. 2009, ApJ, 697, 1071 Atwood, W. B., Albert, A., Baldini, L., et al. 2013, arXiv:1303.3514

Atwood, W. B., Ziegler, M., Johnson, R. P., \& Baughman, B. M. 2006, ApJL, 652, L49

Barr, E. D., Guillemot, L., Champion, D. J., et al. 2013, MNRAS, 429, 1633

Beran, R. J. 1969, Ann. Math. Stat, 40, 1196

Bickel, P., Kleijn, B., \& Rice, J. 2008, ApJ, 685, 384

Caraveo, P. A. 2014, ARA\&A, 52, 211

de Jager, O. C., Raubenheimer, B. C., \& Swanepoel, J. W. H. 1989, A\&A, 221,180

Edwards, R. T., Hobbs, G. B., \& Manchester, R. N. 2006, MNRAS, 372, 1549 
Feroz, F., Hobson, M. P., Cameron, E., \& Pettitt, A. N. 2013, arXiv:1306.2144 Green, D. A. 2014, BASI, 42, 47

Guillemot, L., Johnson, T. J., Venter, C., et al. 2012, ApJ, 744, 33

Jiang, B., Chen, Y., Wang, J., et al. 2010, ApJ, 712, 1147

Kerr, M. 2011, ApJ, 732, 38

Laffon, H., Smith, D. A., \& Guillemot, L. 2015, (for the Fermi-LAT Collaboration), arXiv:1502.03251

Lee, K. J., Guillemot, L., Yue, Y. L., Kramer, M., \& Champion, D. J. 2012, MNRAS, 424, 2832

Lorimer, D., \& Kramer, M. 2005, Handbook of Pulsar Astronomy (Cambridge: Cambridge Univ. Press)

Marelli, M., De Luca, A., \& Caraveo, P. A. 2011, ApJ, 733, 82

Nolan, P. L., Abdo, A. A., Ackermann, M., et al. 2012, ApJS, 199, 31

Pletsch, H. J., \& Clark, C. J. 2014, ApJ, 795, 75
Pletsch, H. J., Guillemot, L., Allen, B., et al. 2012a, ApJ, 744, 105

Pletsch, H. J., Guillemot, L., Allen, B., et al. 2012b, ApJL, 755, L20

Pletsch, H. J., Guillemot, L., Allen, B., et al. 2013, ApJL, 779, L11

Ravi, V., Manchester, R. N., \& Hobbs, G. 2010, ApJL, 716, L85

Ray, P. S., Kerr, M., Parent, D., et al. 2011, ApJS, 194, 17

Romani, R. W. 2012, ApJL, 754, L25

Safi-Harb, S., Dubner, G., Petre, R., Holt, S. S., \& Durouchoux, P. 2005, ApJ, 618,321

Saz Parkinson, P. M., Dormody, M., Ziegler, M., et al. 2010, ApJ, 725, 571

Stroh, M. C., \& Falcone, A. D. 2013, ApJS, 207, 28

Xing, Y., \& Wang, Z. 2014, PASJ, 66, 72

Yamaguchi, H., Badenes, C., Foster, A. R., et al. 2015, ApJL, 801, L3

Yu, M., Manchester, R. N., Hobbs, G., et al. 2013, MNRAS, 429, 688 